\title{
Attack Trees vs. Fault Trees: Two Sides of the Same Coin from Different Currencies
}

\author{
Carlos E. Budde ${ }^{1(\bowtie)}\left(\mathbb{0}\right.$, Christina Kolb ${ }^{1}\left(\mathbb{0}\right.$, and Mariëlle Stoelinga ${ }^{1,2}(\mathbb{C}$ \\ 1 Formal Methods and Tools, University of Twente, Enschede, The Netherlands \\ \{c.e.budde, c.kolb,m.i.a.stoelinga\}@utwente.nl \\ 2 Department of Software Science, Radboud University, Nijmegen, The Netherlands
}

\begin{abstract}
This work compares formal approaches to define and operate with attack trees and fault trees. We start by investigating similarities between the syntactic structure, semantics, and qualitative analysis, of static attack trees and fault trees. Then we point out differences of the analysis methods and metrics between the two formalisms, providing a deeper insight for their dynamic variants. Finally, we overview several extensions and categorise them using the new concept of dimension, which allows us to compare these extensions and point out research gaps.
\end{abstract}

\section{Introduction}

Attack trees (ATs) and fault trees (FTs) are popular formalisms that support the identification, documentation, and analysis of security (resp. safety) risks. They are part of system-engineering frameworks such as SysML-Sec [28], and count with commercial tools such as Isograph's AttackTree and FaultTree+ $[14,15]$.

The popularity of these formalisms in industry is due to their capacity to represent complex processes succinctly and to the desired level of detail. In AT (resp. FT) analysis, a hierarchical diagram is designed to systematically map security (resp. safety) hazards. The resulting model gives insight into the vulnerabilities of the system, which can then be countered cost-efficiently $[18,34]$.

The Origins. This analogous procedural approach is no coincidence: ATs were inspired on FTs. The latter were introduced in 1961 at Bell Labs to study ballistic missiles [30,33]. In 1990 FT analysis was "about 39 years old, and has become a well-recognized tool worldwide" [9]. In contrast, Weiss introduced threat logic trees - the origin of ATs - in 1991, and its "similarity... to fault trees suggests that graph-based security modelling has its roots in safety modelling" [21].

Ever since, these formalisms increased their modelling and analysis power to best satisfy the needs of the safety or security application domain. This has separated the syntax and semantics of new FT- and AT-based formalisms, in spite of

This work was partially funded by NWO project 15474 (SEQUOIA) and ERC Consolidator Grant 864075 (CAESAR).

(C) Springer Nature Switzerland AG 2021

A. Abate and A. Marin (Eds.): QEST 2021, LNCS 12846, pp. 457-467, 2021.

https://doi.org/10.1007/978-3-030-85172-9_24 
their sharing the modelling principle of top-down hierarchical decomposition. In this work we study this disjoint evolution from the perspective of formal methods. We first show in Sect. 2 that the syntax of their so-called static versions, as wells as their corresponding semantics and qualitative analysis, are mathematically equivalent. The only distinction between static FTs and ATs as a formalism is their domain ontology: safety vs. security. This is the root of their subsequent differentiation, which we study in Sect. 3. To compare their extensions in a systematic manner we introduce the notion of dimension, which allows us to contrast parallel (even symmetrical) evolutions. The work concludes in Sect. 4.

Formalism. A formalism is defined by 1. an (unambiguous) syntax to represent its elements, called models; 2. a semantics that maps each model to a (unique) mathematical object; 3. a domain ontology in which the models are interpreted. The three parts of this preliminary definition are formalised in the sequel.

Related Work. Surveys on FTs are [18] and [30]. The latter covers modelling and analysis tools. The former pinpoints limitations of FTs to assess the reliability of static systems (only), and mentions extensions that overcome them, e.g. dynamic FTs [8], state-event FTs [19], and Stochastic Hybrid FT Automata [6]. Standard FT analysis and its extensions are also extensively discussed in [30], including technical details of different FT models and analyses.

Surveys on attack trees, [21] and [34], present the state of the art in graphicalhierarchical attack/defense modelling. The latter is a modern and comprehensive summary on the use of formal methods to enhance security evaluation. It references software tools, and discusses steps for industrial technology transfer.

\section{Similarities Between Fault and Attack Trees}

ATs and FTs follow the same modelling principle: an expert panel identifies a main event of interest - one top element - and refine it logically to the level of well-understood basic components or actions - the set of basic elements - $[21,30]$. This analogous model-building process results in identical syntactic structures.

\subsection{Syntactic Structure: Static FTs and ATs}

The vanilla version of FTs and ATs, so-called static fault or attack trees, have the same syntax. We unify them under the concept of logical-decomposition tree.

Definition 1 (LDT). A logical-decomposition tree (LDT for short) is a tuple $T=\langle N, t$, ch $\rangle$ where: (i) $N$ is a finite set of nodes; (ii) $t: N \rightarrow\{A N D, O R$, $L E A F\}$ gives the type of each node; (iii) ch: $\rightarrow 2^{N}$ gives the (possibly empty) set of children of a node. Moreover, $T$ satisfies the following constraints: (a) $\langle N, E\rangle$ is a connected directed acyclic graph (DAG), where $E=$ $\left\{(v, u) \in N^{2} \mid u \in \operatorname{ch}(v)\right\} ;$ (b) T has a unique root, denoted $R_{T}: \exists ! R_{T} \in N . \forall v \in$ N. $R_{T} \notin \operatorname{ch}(v) ;(\mathrm{c}) L E A F$ nodes $N_{L} \subseteq N$ are the leaves of $T: \forall v \in N$. $t(v)=L E A F \Leftrightarrow v \in N_{L} \Leftrightarrow \operatorname{ch}(v)=\varnothing$. 
So, syntactically, static FTs and ATs are LDTs (VOT gates in static FTs are syntactic sugar of AND and OR gates). LDTs can be proper trees or DAGs: the difference is that in proper trees, each child node has exactly one parent. Node $v \in N$ is the parent of $u \in N$ iff $u$ is a child of $v$, i.e. $T$ has the edge $v \rightarrow u$. By definition, $L E A F$ nodes are not parents: parent nodes are called gates.

Logical Gates. An LDT represents the logical decomposition of events via disjunction and conjunction, which can be interpreted as a Boolean function. Consider e.g. a wooden gate that can break due to rotten wooden planks or rusty hinges (or both); and the hinges rust if they are made of iron AND the environment is humid AND sufficient time elapses. This decomposition is safety-oriented. From an analogous security perspective, the wooden gate is breached by dislodging the hinges oR cracking the wooden planks, and hinges can be dislodged if the alloy is fragile AND the attacker has a crowbar AND enough strength. Both cases yield the LDT $\left\langle\left\{a, b, c, d, g_{1}, g_{2}\right\}, t, c h\right\rangle$ with leaves $N_{L}=\{a, b, c, d\}$ and gates $t\left(g_{1}\right)=A N D, t\left(g_{2}\right)=O R$, s.t. $\operatorname{ch}\left(g_{1}\right)=\left\{a, g_{2}\right\}, \operatorname{ch}\left(g_{2}\right)=\{b, c, d\}$. This represents the Boolean function $\lambda a b c d . a \vee(b \wedge c \wedge d)$ whose atoms take safety or security meaning: this is denoted $\operatorname{OR}(a, \operatorname{AND}(b, c, d))$ and visualised as Fig. 1a.

Visualisation. FTs and ATs are graphical formalisms, drawn as in Fig. 1 with the root on top, and every child connected to an (upper) parent by a line. Leaves are circles, and logical gates resemble their electronic-circuit counterparts.

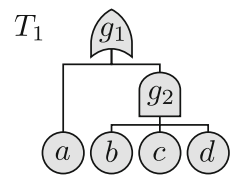

(a) $\operatorname{OR}(a, \operatorname{AND}(b, c, d))$

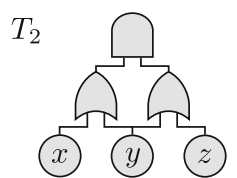

(b) $\operatorname{AND}(\operatorname{OR}(x, y), \operatorname{OR}(y, z))$

Fig. 1. $T_{1}$ has tree structure; the or gate $g_{1}$ is the root; $g_{2}$ is an AND gate. $T_{2}$ has DAG structure: $y$ has two parents.

\subsection{Semantics and Analysis}

Once an LDT model $T$ has been created, it is studied to find safety/security vulnerabilities of the system. For this, $T$ is bestowed with formal semantics.

Structure Function. These semantics can be given via a function $f_{T}: 2^{N_{L}} \rightarrow \mathbb{B}$ that indicates whether a set of basic elements trigger the top element of $T$. That is, $f_{T}(A)=\top$ iff the Boolean function represented by $T$ is satisfied by the mapping $(A \mapsto \top) \cup\left(\left(N_{L} \backslash A\right) \mapsto \perp\right)$, where \denotes set difference. For instance for $T_{1}$ in Fig. 1a, to evaluate $f_{T_{1}}(\{a, c\})$ one maps $a$ and $c$ to $\top, b$ and $d$ to $\perp$, and evaluates the Boolean function represented by $T_{1}-\lambda a b c d \cdot a \vee(b \wedge c \wedge d)-$ which returns $T$. This so-called structure function $f_{T}$ is given by the syntactic structure of $T$, and hence it is analogous for static FTs and ATs [17,30]. 
Evidence. The set $A \subseteq N_{L}$ on which $f_{T}$ is evaluated is called evidence: for ATs it represents the steps carried out by an attacker; for FTs it represents elements that have failed. If $f_{T}(A)=\top$ then $A$ is called valid evidence; else it is invalid. Valid evidence $A$ is called minimal if no proper subset of $A$ is valid. For instance in Fig. $1,\{a\}$ and $\{x, z\}$ are minimal evidence of $T_{1}$ and $T_{2}$ resp., $\{a, b\}$ is also valid (but not minimal) evidence, and $\{x\}$ is invalid. In FT analysis, minimal evidence is also called "minimal cut set" or "prime implicant."

Formal Semantics. Static FTs and ATs are coherent: adding basic elements to evidence preserves its validity [3]. That is, if $f_{T}(A)=\top$, then $f_{T}(A \cup\{a\})=\top$ for every $a \in N_{L}$. Thus, all valid evidence of $T$-i.e. that can trigger its top element - is characterised by the collection of minimal evidence. This gives rise to the formal semantics of $T: \llbracket T \rrbracket=\left\{A \subseteq N_{L} \mid f_{T}(A)=\top \wedge A\right.$ is minimal $\} .{ }^{1}$

Qualitative Analysis. Since $\llbracket T \rrbracket$ subsumes all ways to trigger the top element of $T$, its computation provides key information on the vulnerability of the system. For FTs, any $A \in \llbracket T \rrbracket$ with few elements pinpoints a safety hazard-where a few basic failures can trigger a system-level failure - and likewise for ATs. The amount of subsets in $\llbracket T \rrbracket$ can be exponential on the number of nodes in $T$ [24]. Since this hinders computations, and given the interest in small sets from $\llbracket T \rrbracket$, FT analysis sometimes bounds the size of the minimal evidence to compute [32].

Thus, static FTs and ATs are mathematically equivalent: their syntax is given by an LDT, $T$, and their semantics by the set of minimal evidence, $\llbracket T \rrbracket$. What sets them apart as formalisms is their domain ontology, i.e. their application domain: safety and security have different goals, fulfilled by enriching LDTs with (a) attributes on the leaves, and (b) new types of gates. In Sect. 3 we show how this is the root of several differences between FTs and ATs.

\section{Differences Between Fault and Attack Trees}

The aforementioned similarities apply only to static FTs and ATs. Later extensions to these formalisms, e.g. to include notions of complement and dynamic behaviour, have caused them to grow in different directions. We discuss this in Sect.3.2 but first we show, in Sect.3.1, that the different goals of the safety/security domains break the analogies even for static FTs and ATs.

\subsection{Analyses that Differ for Static FTs and ATs}

Quantitative Analysis. Beyond the constitution of each set $A \in \llbracket T \rrbracket$, it is useful to quantify their relevance. For example, if every basic element $a_{i}$ has a probability $p_{i} \in[0,1]$ of occurrence, one can compute the total probability of some evidence $A$ [26]. Similarly, values $\lambda_{i} \in \mathbb{R}_{>0}$ can describe the rate at which these basic probabilities increase with time. Then one can measure the system unreliability, i.e. the probability of triggering the top element at various mission

\footnotetext{
${ }^{1}$ There are other equivalent ways to define static AT/FT semantics, e.g. bundles [25].
} 
times. Rates also enable time-only measurements, such as the mean time it takes for some evidence $A \in \llbracket T \rrbracket$ to be observed [30].

All these quantitative queries, that deal with the probability and frequency of occurrence of events, are characteristic of FT analysis [9,24,30,32]. The reason is that it is feasible and useful to estimate e.g. the mean time to failure (MTTF) of machine components: this allows engineers to compute safe, cost-optimal policies for inspection, maintenance, and replacement of company assets [29].

In contrast, the probability of basic attacks in ATs are very hard to know [11]. Unknown vulnerabilities may increase it, also its frequency, and the conditional probability tables are usually not-knowable. Therefore, it is typical to query the max (rather than total) attack probability [34]. This is also cost-driven: rather than try everything, an attacker may only choose the most promising attack.

The time for an attack is also described differently than for a failure: whereas failures typically have MTTF or rate values, basic attacks steps can be given [min, max] intervals, and further differentiate activation from execution time [23].

Finally, quantitative analyses in ATs can be richer than in FTs, exploring attribute domains beyond time and probability. These include the cost to carry out certain attacks, the skill or psychological profile required to do it, the max damaged caused, and Pareto analyses thereof $[2,11,23]$.

\section{Propagation of Values Through Logical Gates.}

When the AT or FT is a proper tree, quantitative queries can be computed bottom-up directly on its syntactic structure. For this, the values of the basic elements are propagated upwards in the tree [25]. For instance if basic elements $a$ and $b$ cost resp. $€ 3$ and $€ 7$, then the cost of $\operatorname{Or}(a, b)$ is the $\min , € 3$, and the cost of $\operatorname{AND}(a, b)$ is the sum, $€ 10$.

However, here too we find a remarkable difference between static FTs and ATs, that is overlooked by

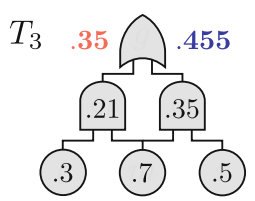

Fig. 2. Probability computation: AT (left, red) vs. FT (right, blue) (Color figure online) many reviews in spite of its apparent impact in quantitative analyses. In FTs, the "probability of failure" asks for total probability, so the (probability) value of an or gate is the sum of the values of its children, minus the value of their intersection. Instead and as indicated above, attacks are characterised by their max probability, so the value of an oR gate in an AT is the max value among its children. This is illustrated in Fig. 2: the values in the basic elements are given; the probability of an AND gate is the product of its children; but if $T_{3}$ is an AT, its ( $\max$ ) attack probability is .35; and if it is an FT, its (total) failure probability is .455 .

This different propagation of values also affects conjunctive gates (AND), most notably with time attributes. The basic elements in static FTs refer to failures in components, which are under simultaneous use and therefore whose degradation is concurrent. Thus the MTTF of an AND gate is the max across the MTTF of its children. ATs, in contrast, have more ways to describe a conjunction of activities. 
Table 1. Overview of extensions to the FT and AT formalisms

\begin{tabular}{|c|c|c|c|c|}
\hline \multicolumn{4}{|c|}{ Form. Extensions } & \multirow{2}{*}{$\begin{array}{l}\text { Main features } \\
\text { FTS }+ \text { PAND }+ \text { SPARE }+ \text { FDEP }\end{array}$} \\
\hline \multirow{4}{*}{ FTs } & DFT & [8] & Dynamic FTs & \\
\hline & RFT & {$[4,7]$} & Repairable FTs & FTs + repair boxes \\
\hline & E-DFT & {$[10]$} & Extended DFTs & DFTs + gen. SPAREs + triggers \\
\hline & SE-FT & {$[27]$} & State/Event FTs & FTs + Petri nets in leaves \\
\hline \multirow{2}{*}{ ATs } & SAND-AT & {$[16]$} & SAND attack trees & ATs with sequential AND \\
\hline & ADTree & {$[20]$} & Attack-defense trees & ATs + defenses \\
\hline \multirow{4}{*}{$\begin{array}{c}\text { FTs } \\
\& \\
\text { ATs }\end{array}$} & BDMP & {$[5]$} & Boolean Markov proc. & FTs + ATs + triggers + repairs \\
\hline & CFT & {$[31]$} & Component FTs & ATs + FTs with modular structure \\
\hline & AFT & {$[22]$} & Attack-Fault Trees & SAND-ATS + DFTS \\
\hline & FT-AT & {$[12]$} & FTs integrated to ATs & FTS whose BEs are refined as ATS \\
\hline
\end{tabular}

In particular they could require time-exclusion: consider one attacker who must perform multiple actions, e.g. deactivating an alarm and silencing the dog. Here, the time to execute all attacks is not the max, but the sum of the times [1]. ATs can indicate this with a new gate: sequential-AND (SAND).

Generally speaking, static ATs and FTs have begotten independent extensions that introduce new gates. For instance, SEQ enforcers in FTs can be seen as analogous to SAND gates in ATs. In general, however, these extensions further differentiate the AT and FT formalisms, as we discuss in Sect. 3.2.

\subsection{Extensions of the Formalisms}

So far we considered (only) static FTs and ATs, pointing out their similarities and differences. In this section we revise several extensions that grow beyond them. We first overview some prominent formalisms in Table 1, and then refine the comparison by defining and making use of the concept of dimension.

Safety Extensions of FTs. The first formalisms in Table 1 are important extensions of FTs: DFTs are static FTs plus PAND gates (that fail if all children fail in left-to-right order), SPARE gates (for spare parts), and FDEPs (that model common-cause failures); RFTs are static FTs with repair boxes, that can repair failed BEs; and E-DFTs are generalised DFTs with triggers, which allow arbitrary subtrees as spares, and whose FDEPs can trigger gates as well as BEs.

FTs + Security. While DFTs, RFTs, and E-DFTs, improve safety modelling in fault trees, other extensions can cover security aspects. For instance, SE-FTs have Petri nets as basic elements. These are more versatile than the usual twostate BEs, allowing state changes that can model safety and security hazards.

Security Extension of ATs. There also exist extensions to improve security modelling of attack trees: $S A N D-A T s$ add dynamic behaviour to ATs, by forc- 
ing attacks to occur in a specific order via SAND gates ${ }^{2}$; and ADTrees model protections against attacks via special defense nodes.

Combinations of FTs and ATs. All those formalisms extend either FTs or ATs, but there also exist formalisms that combine them. BDMPs can have ATs as subtrees of FTs and vice versa, and allow propagations of failures/attacks (and repairs) via triggers among gates. CFTs add modular FTs to ATs, to foster large-system analysis via decoupled studies of smaller components. In contrast, AFTs trade scalability for versatility, by merging DFTs (with all its dynamic gates) with ATs plus SAND gates. Finally, FT-ATs refine the BEs in fault trees via attack trees, modelling attackers that try to force a system failure.

Note that, interestingly and to the best of our knowledge, no formalism that combines FTs with ATs includes defenses. More importantly, we find independent extensions that overlap in some modelling goals, e.g. RFTs and the repairs of BDMPs. We compare these (partial) overlaps via dimensions.

Dimensions. An extension augments the modelling power of FTs/ATs. Some extensions reach to each other, e.g. AFTs and BDMPs are in both domain ontologies (safety and security). But other extensions are parallel: compare DFTs to SAND-ATs, both of which make the order of events relevant but without crossing the safety/security line. We thus identify different ways to classify the space of formalisms, where a dimension $d$ defines (not necessarily exclusive) classes that are orthogonal to those defined by another dimension $d^{\prime}$. For example, the domain ontology can be seen as a domain dimension: it defines the classes safety and security s.t. the formalisms $\{$ FT, DFT $\}$ are in safety, $\{$ AT,SAND-AT $\}$ are in security, and $\{\mathrm{AFT}, \mathrm{BDMP}\}$ are in both. Further dimensions to classify formalisms include dynamics - the order of events matters or not-and complement - there is a single type of event (e.g. attacks), or complementary types (also defenses). Figure 3 shows a scheme of this 3D classification.

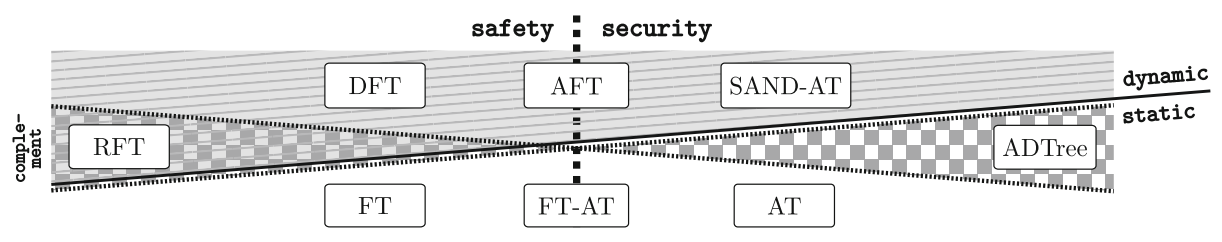

Fig. 3. Dimensional split of formalisms: domain, dynamics, and complement.

Such concept of dimension resembles that of an ontology in information science [13]. For us, different dimensions yield orthogonal classifications of the same set of individuals. These individuals are the formalisms within scope: we use Table 1 as the scope, but Definition 2 generalises to any FT/AT extension.

\footnotetext{
${ }^{2}$ SANDS in ATs force a sequence of events, similarly to SEQ enforcers in certain flavours of FTs; this differs from PAND gates in DFTs, which observe the order of events.
} 
Table 2. Dimensional split of formalisms in Table 1

\begin{tabular}{|c|c|c|c|c|c|c|c|c|c|c|c|c|c|}
\hline \multicolumn{2}{|c|}{ Dimension } & FT & DFT & RFT & E-DFT & SE-FT & BDMP & CFT & AFT & FT-AT & SAND-AT & ADTree & $\mathrm{AT}$ \\
\hline ह & safety & $\checkmark$ & $\checkmark$ & $\checkmark$ & $\checkmark$ & $\checkmark$ & $\checkmark$ & $\checkmark$ & $\checkmark$ & $\checkmark$ & & & \\
\hline 8 & security & & & & & $\checkmark$ & $\checkmark$ & $\checkmark$ & $\checkmark$ & $\checkmark$ & $\checkmark$ & $\checkmark$ & $\checkmark$ \\
\hline$\dot{\varepsilon}$ & static & $\checkmark$ & & & & & & $\checkmark$ & & $\checkmark$ & & $\checkmark$ & $\checkmark$ \\
\hline శో & dynamic & & $\checkmark$ & $\checkmark$ & $\checkmark$ & $\checkmark$ & $\checkmark$ & & $\checkmark$ & & $\checkmark$ & & \\
\hline हुं & $\begin{array}{r}\text { single } \\
\text { dual }\end{array}$ & $\checkmark$ & $\checkmark$ & $\checkmark$ & $\checkmark$ & $\checkmark$ & $\checkmark$ & $\checkmark$ & $\checkmark$ & $\checkmark$ & $\checkmark$ & $\checkmark$ & $\checkmark$ \\
\hline
\end{tabular}

Definition 2 (Dimension). A dimension is an ontology with two or more nonempty classes, whose individuals are the formalisms from Table 1. A dimensional base $\mathbb{D}=\left\{d_{i}\right\}_{i=1}^{n}$ is a finite set of orthogonal dimensions.

So far we have compared formalisms exclusively from the perspective of the domain dimension: we now turn our attention to dynamics and complement.

Note, however, that these three dimensions - that Table 2 defines in our full scope - are not exhaustive. We identify at least an extra complexity dimension, sensitive to the number of states of the basic elements. In terms of complexity, FTs and ATs are simple (binary states), while SE-FTs and Fault Maintenance Trees [29] are complex (its basic elements are resp. Petri nets and Erlang chains).

Dynamics. This dimension classifies formalisms based on whether their semantics caters for order. The broadest possible classes are static and dynamic. The success of the top element in a static formalism does not depend on the order in which the basic elements occur. This includes FTs, ATs, CFTs, AT-FTs, and ADTrees. Other formalisms in Table 1 are dynamic: they either enforce an order, e.g. SAND gates and SEQ enforcers; or the propagation of success in some gates depends on it, e.g. PAND gates in DFTs. Besides a richer semantics (that affects qualitative analyses), dynamic formalisms have more complex quantitative analyses. In a static AT, the attack time of a conjunctive gate is the max time among its children. Instead, in a SAND-AT, it is the max or the sum of the times, depending on whether the gate is a "parallel" AND or a sequential-AND.

Complement. This dimension has two classes: dual formalisms have two complementary type of events; single formalisms have one. By event we mean a change of state, whose multiplicity can have syntactic support via a type system, or it can reside entirely at semantic level. An example of the latter are repairs in RFTs: their (single-typed) basic elements can transit in both directions between their active and failed states. An example of dual events via types are attack- vs. defense-nodes in ADTrees: given an attack, if the counter-defense occurs, then the state of the corresponding gate changes first to "attacked" and then to "not attacked." This differs from the absence of an attack for quantitative queries, e.g. to compute attack cost. In Table 1, the only formalisms in the dual class of this complement dimension are ADTree, RFT, and BDMP. All the rest are single: only one change of state can happen, namely a failure (resp. an attack) that involves a transition from an active to a failed (resp. attacked) state. 
Finally, we note that comparing the classification of different dimensions helps to spot research gaps. For instance, from the five formalisms in both classes of the domain dimension, only BDMPs are dual as per complement. Since that comes from repairs of failed basic elements, we know that no formalism in Table 1 that combines safety and security includes defenses, as pointed out earlier.

\section{Conclusions and Future Work}

We have compared FTs against ATs, showing how they model system vulnerabilities in the same mathematical static way. However, their different domain ontologies - safety for FTs, security for ATs - gives rise to different quantitative analyses. This shows in the algebra used to propagate values through gates, e.g. to compute the probability of a failure vs. that of an attack. Moreover, new gates have been added to FTs and ATs, extending these formalisms in directions that sometimes cross each other. We introduced the concept of dimension to classify these extensions, thus generalising the safety/security dichotomy.

These studies can be deepened by finding new dimensions to compare formalisms. Our dimensional split offers a high-level view that helps to spot research gaps. In particular, we found no formalism that merges ATs and FTs, that also includes defenses against attacks. Neither have we found formalisms with clearlydifferentiated AT/FT submodules, such as FT-ATs, that also offer dynamic gates and repairs, such as BDMPs. The industrial relevance of model visualisation, plus the need for versatile modelling, makes this gap a promising line of research.

\section{References}

1. Arnold, F., Hermanns, H., Pulungan, R., Stoelinga, M.: Time-dependent analysis of attacks. In: Abadi, M., Kremer, S. (eds.) POST 2014. LNCS, vol. 8414, pp. 285-305. Springer, Heidelberg (2014). https://doi.org/10.1007/978-3-642-547928 _ 16

2. Aslanyan, Z., Nielson, F.: Pareto efficient solutions of attack-defence trees. In: Focardi, R., Myers, A. (eds.) POST 2015. LNCS, vol. 9036, pp. 95-114. Springer, Heidelberg (2015). https://doi.org/10.1007/978-3-662-46666-7_6

3. Barlow, R.E., Proschan, F.: Statistical Theory of Reliability and Life Testing: Probability Models. International Series in Decision Processes. Holt, Rinehart and Winston, New York (1975)

4. Bobbio, A., Codetta-Raiteri, D.: Parametric fault trees with dynamic gates and repair boxes. In: RAMS, pp. 459-465. IEEE (2004). https://doi.org/10.1109/ RAMS.2004.1285491

5. Bouissou, M.: BDMP (Boolean logic Driven Markov Processes) as an alternative to Event Trees. In: ESREL 2008 (2008)

6. Chiacchio, F., D’Urso, D., Compagno, L., Pennisi, M., Pappalardo, F., Manno, G.: SHyFTA, a stochastic hybrid fault tree automaton for the modelling and simulation of dynamic reliability problems. Expert Syst. Appl. 47, 42-57 (2016). https://doi. org/10.1016/j.eswa.2015.10.046 
7. Codetta-Raiteri, D., Iacono, M., Franceschinis, G., Vittorini, V.: Repairable fault tree for the automatic evaluation of repair policies. In: DSN, pp. 659-668. IEEE Computer Society (2004). https://doi.org/10.1109/DSN.2004.1311936

8. Dugan, J., Bavuso, S., Boyd, M.: Fault trees and sequence dependencies. In: ARMS, pp. 286-293. IEEE (1990). https://doi.org/10.1109/ARMS.1990.67971

9. Ericson, C.A.: Fault tree analysis - A history. In: 17th International System Safety Conference, pp. 1-9 (1999)

10. Arnold, F., Belinfante, A., Van der Berg, F., Guck, D., Stoelinga, M.: DFTCalc: a tool for efficient fault tree analysis. In: Bitsch, F., Guiochet, J., Kaâniche, M. (eds.) SAFECOMP 2013. LNCS, vol. 8153, pp. 293-301. Springer, Heidelberg (2013). https://doi.org/10.1007/978-3-642-40793-2_27

11. Fila, B., Wideł, W.: Attack-defense trees for abusing optical power meters: a case study and the OSEAD tool experience report. In: Albanese, M., Horne, R., Probst, C.W. (eds.) GraMSec 2019. LNCS, vol. 11720, pp. 95-125. Springer, Cham (2019). https://doi.org/10.1007/978-3-030-36537-0_6

12. Fovino, I.N., Masera, M., De Cian, A.: Integrating cyber attacks within fault trees. Reliab. Eng. Syst. Saf. 94(9), 1394-1402 (2009). https://doi.org/10.1016/j.ress. 2009.02.020

13. Guarino, N.: Formal ontology, conceptual analysis and knowledge representation. Int. J. Hum.-Comput. Stud. 43(5), 625-640 (1995). https://doi.org/10.1006/ijhc. 1995.1066

14. Isograph: AttackTree. https://www.isograph.com/software/attacktree/

15. Isograph: FaultTree+. https://www.isograph.com/software/reliabilityworkbench/fault-tree-analysis-software/fault-tree-analysis/

16. Jhawar, R., Kordy, B., Mauw, S., Radomirović, S., Trujillo-Rasua, R.: Attack trees with sequential conjunction. In: Federrath, H., Gollmann, D. (eds.) SEC 2015. IAICT, vol. 455, pp. 339-353. Springer, Cham (2015). https://doi.org/10.1007/ 978-3-319-18467-8 23

17. Jürgenson, A., Willemson, J.: Computing exact outcomes of multi-parameter attack trees. In: Meersman, R., Tari, Z. (eds.) OTM 2008. LNCS, vol. 5332, pp. 1036-1051. Springer, Heidelberg (2008). https://doi.org/10.1007/978-3-540-8887348

18. Käbir, S.: An overview of fault tree analysis and its application in model based dependability analysis. Expert Syst. Appl. 77, 114-135 (2017). https://doi.org/10. 1016/j.eswa.2017.01.058

19. Kaiser, B., Gramlich, C., Förster, M.: State/event fault trees-a safety analysis model for software-controlled systems. Reliab. Eng. Syst. Saf. 92(11), 1521-1537 (2007). https://doi.org/10.1016/j.ress.2006.10.010

20. Kordy, B., Mauw, S., Radomirović, S., Schweitzer, P.: Foundations of attackdefense trees. In: Degano, P., Etalle, S., Guttman, J. (eds.) FAST 2010. LNCS, vol. 6561, pp. 80-95. Springer, Heidelberg (2011). https://doi.org/10.1007/978-3642-19751-2 6

21. Kordy, B., Piètre-Cambacédès, L., Schweitzer, P.: DAG-based attack and defense modeling: don't miss the forest for the attack trees. Comput. Sci. Rev. 13-14, 1-38 (2014). https://doi.org/10.1016/j.cosrev.2014.07.001

22. Kumar, R., Stoelinga, M.: Quantitative security and safety analysis with attackfault trees. In: 18th International Symposium on HASE, pp. 25-32 (2017)

23. Kumar, R., Ruijters, E., Stoelinga, M.: Quantitative attack tree analysis via priced timed automata. In: Sankaranarayanan, S., Vicario, E. (eds.) FORMATS 2015. LNCS, vol. 9268, pp. 156-171. Springer, Cham (2015). https://doi.org/10.1007/ 978-3-319-22975-1 11 
24. Lee, W., Grosh, D., Tillman, F., Lie, C.: Fault tree analysis, methods, and applications - a review. IEEE Trans. Reliab. R-34(3), 194-203 (1985). https://doi.org/ 10.1109/TR.1985.5222114

25. Mauw, S., Oostdijk, M.: Foundations of attack trees. In: Won, D.H., Kim, S. (eds.) ICISC 2005. LNCS, vol. 3935, pp. 186-198. Springer, Heidelberg (2006). https:// doi.org/10.1007/11734727_17

26. Rauzy, A.: New algorithms for fault trees analysis. Reliab. Eng. Syst. Saf. 40(3), 203-211 (1993). https://doi.org/10.1016/0951-8320(93)90060-C

27. Roth, M., Liggesmeyer, P.: Modeling and analysis of safety-critical cyber physical systems using state/event fault trees. In: SAFECOMP (2013)

28. Roudier, Y., Apvrille, L.: SysML-Sec: a model driven approach for designing safe and secure systems. In: MODELSWARD, pp. 655-664. IEEE (2015)

29. Ruijters, E., Guck, D., Drolenga, P., Peters, M., Stoelinga, M.: Maintenance analysis and optimization via statistical model checking. In: Agha, G., Van Houdt, B. (eds.) QEST 2016. LNCS, vol. 9826, pp. 331-347. Springer, Cham (2016). https:// doi.org/10.1007/978-3-319-43425-4_22

30. Ruijters, E., Stoelinga, M.: Fault tree analysis: a survey of the state-of-the-art in modeling, analysis and tools. Comput. Sci. Rev. 15-16, 29-62 (2015). https://doi. org/10.1016/j.cosrev.2015.03.001

31. Steiner, M., Liggesmeyer, P.: Combination of safety and security analysis - finding security problems that threaten the safety of a system (2016)

32. Vesely, W., Stamatelatos, M., Dugan, J., Fragola, J., Minarick, J., Railsback, J.: Fault tree handbook with aerospace applications. NASA Office of Safety and Mission Assurance, version 1.1 (2002)

33. Watson, H.: Launch control safety study. Techical report Section VII, Vol. 1, Bell Labs (1961)

34. Wideł, W., Audinot, M., Fila, B., Pinchinat, S.: Beyond 2014: formal methods for attack tree-based security modeling. ACM Comput. Surv. 52(4) (2019). https:// doi.org/10.1145/3331524 